\title{
Die sozialpädagogische Schule als Präventionsmodell für abweichendes Verhalten
}

\section{A escola sociopedagógica como modelo de prevenção para comportamento não-conformado}

\author{
Eric Mührel \\ Instituto de Sistemas Sociais no Campus Emden (Alemanha) \\ Fachhochschule Oldenburg, Emden (Deutschland)
}

\section{Tradução de Vera Herweg Westphal}

\section{Die sozialpädagogische Schule als Präventionsmodell für abweichendes Verhalten}

Zusammenfassung: Dieser Aufsatz betrachtet die Schule als sozialpädagogischen Raum, um Demokratie zu erlernen und zu erfahren. Dadurch kann deviantem Verhalten und Gewalt vorgebeugt werden. Zunächst wird das sozialpädagogische Modell von Dewey dargestellt. Nach Dewey sollte der Schüler mittels der Erfahrung lernen. Der Schüler lernt Probleme zu analysieren und in der Gruppe zu lösen. In der Folge werden das Verhältnis zwischen sozialer Integration und Desintegration und die Möglichkeiten des Handels der sozialpädagogischen Schule in diesem Kontext dargestellt, wobei die soziale und emotionale Annerkenung als Gewaltprävention angesehen wird. Zuletzt wird die Expansion der Profession Sozialarbeit und Sozialpädagogik in den Schulen betrachtet, indem diese mit Gewaltund Konfliktprävention intervenieren.

Schlüsselwörter: Sozialpädagogik, Sozialarbeit in der Schule, sozialpädagogische Schule, Gewaltprävention, Soziale AntiGewaltprogramme.

\section{A escola sociopedagógica como modelo de prevenção para comportamento não-conformado}

Resumo: Este artigo pondera sobre a escola como espaço social e pedagógico para aprender democracia e prevenir comportamentos desviantes, atitudes não conformes, enfim, prevenir violência. Assim aborda a redescoberta da escola a partir da proposta de Dewey, para o qual esta deveria proporcionar o aprendizado por meio de experiências, onde o aluno aprenderia a se colocar nas situações reais para tentar resolvê-las coletivamente. Na seqüência, apresenta a relação entre integração e desintegração social e as possibilidades de atuação neste contexto a partir da proposta de uma escola social e pedagógica, enfocando as dimensões do reconhecimento emocional e social em contextos de prevenção à violência. Por último, apresenta as possibilidades de expansão do campo de atuação dos assistentes sociais na realidade escolar.

Palavras-chave: pedagogia social escolar, Serviço Social escolar, escola sociopedagógica, prevenção à violência, programas sociais antiviolência.

\section{The Socio-pedagogical School as a Model to Prevent Non Conformist Behavior}

Abstract: This article analyzes the school as a social and pedagogical space for learning about democracy and preventing deviant behavior and non-conforming attitudes, that is, preventing violence. It thus analyzes the rediscovery of the school from the Dewy proposal, which maintained that learning should occur through experiences, in which students would place themselves in real situations to try to resolve them collectively. It then presents the relationship between social integration and disintegration and the possibilities for action in this context based on the proposal of a social and pedagogic school, focusing on the dimensions of emotional and social recognition in contexts of violence prevention. In conclusion, it discusses possibilities for expansion of the field of action of social workers in the school reality.

Key words: educational social pedagogy, educational Social Work, educational socio-pedagogy, violence prevention, antiviolence social programs. 


\section{Einleitung}

Auf die Kinder und Jugendlichen dieses Landes wartet ein ganzes Arsenal von Möglichkeiten devianten, also abweichenden Verhaltens. Das Risikopotential für die Jugend hinsichtlich primärer wie sekundärer Devianz scheint enorm hoch zu sein, so wird es zumindest in den Medien gern vermittelt. Begriffe wie Medienverwahrlosung, Schulabstinenz und Risikoschüler haben sich dort wie im wissenschaftlichen Diskurs fest etabliert. Gerade auch innerhalb der Schule scheint abweichendes Verhalten normal zu sein. Dafür sprechen schon einige spektakuläre Aufdeckungen von Mobbing, Abziehen und permanenten Demütigungen einzelner Schüler oder gar Gruppen von Schülern in Hauptschulen und berufsbildenden Schulen in den vergangenen Jahren. Tatsächlich aber ist das Risikopotential für eine bestimmte Gruppe von Jugendlichen besonders hoch. Es sind jene mit schlechten Bildungschancen und damit mit mangelnden Möglichkeiten gesellschaftlicher Partizipation. Dazu ein Beispiel: der frühere Bundeswirtschafts - und - arbeitsminister Wolfgang Clement gab im Sommer des Jahres 2005 zu, dass mit den Programmen des Förderns und Forderns im Kontext von Hartz $I V$ nur die Hälfte der anvisierten Jugendlichen und Heranwachsenden erreicht werden könne. Es fragt sich dann, was mit der anderen Hälfte geschieht. Wird sie, verabschiedet aus der Partizipation an der Gesellschaft, nicht besonders gefährdet sein hinsichtlich sekundärem abweichendem Verhalten in all seinen Facetten?

Doch wie wird auf die vermeintliche Erhöhung des Risikopotentials abweichenden Verhaltens seitens der Politik reagiert? Der Kriminologe Christian Pfeiffer konstatiert, dass die Investition in die Jugend die zentrale Leitlinie für die Politik werden muss. Damit verbindet er die Frühförderung von Kindern, den Ausbau von Schulen zu Ganztagsschulen und auch eine Stärkung der Hochschulen. Die Politik verhalte sich aber so, als ob die Zukunft Deutschland im Ausbau von Gefängnissen liege. Er belegt dies mit eindrücklichen Zahlen. So sei trotz günstiger Kriminalitätsentwicklung die Zahl der Strafgefangenen seit 1992 um mehr als $40 \%$ gestiegen. Die Mehrkosten hierfür betragen nach Pfeiffer 5 Milliarden Euro jährlich. Hinzu kämen noch im Bau befindliche Neubauten von Justizvollzugsanstalten in Höhe von 1,4 Milliarden Euro (PFEIFFER, 2005). Der

\section{Introdução}

Existem grandes possibilidades de ocorrerências de comportamentos irregulares por parte de crianças e dos jovens na Alemanha, portanto, de apresentarem condutas que chamem atenção. O potencial de risco para a juventude, tendo em vista o desvio $^{1}$ primário e secundário, parece ser muito grande, pelo menos é o que a mídia tem colocado. Conceitos como abandono, evasão, abstinência escolar e escolares em situação de risco, têm se consolidado tanto no discurso midiático como no científico. Parece que, especialmente na escola, o comportamento divergente e estranho é normal. Isto pode ser visto em notícias sobre o mobbing ${ }^{2}$, a desconsideração e as humilhações permanentes de alguns escolares ou mesmo de grupos de escolares em estabelecimentos de ensino médio ou de ensino profissional nos últimos anos. Mas o potencial de risco é muito alto para um determinado grupo de jovens. São aqueles com poucas possibilidades de formação e, por isso, com reduzidas possibilidades de participação social. Sobre este assunto pode ser citado um exemplo do anterior ministro de Economia e Trabalho, Wolfang Clement, quando admitiu, no verão de 2005, que os programas de fomento no contexto do Hartz IV ${ }^{3}$ só atingiam a metade do número de jovens, ao qual deveria realmente atingir. Com isto, coloca-se a pergunta sobre o que acontece à outra metade dos jovens. Será que essa camada, excluída da participação social, não corre especialmente riscos em relação a comportamentos secundários desviantes em todas as suas facetas?

Entretanto, como a política tem reagido à elevação do potencial de risco de comportamento desviante? O estudioso de crimes, Christian Pfeiffer, constata que o investimento na juventude deve se tornar a principal diretriz da política. Para ele, isto abrange o estímulo precoce de crianças, a ampliação do período escolar, tornando-o de tempo integral, bem como o fortalecimento das escolas de nível superior. Ao invés disso, a política tem se direcionado de tal forma, como se o futuro da Alemanha estivesse na ampliação de prisões. Ele comprova isto com números impressionantes, quando mostra que, apesar de um controle de criminalidade, desde 1992 o número de presos cresceu em mais de $40 \%$, representando gastos adicionais que chegam a mais de cinco bilhões de euros por ano. Acresce-se ainda, a construção de novas instituições de execução de 
Ausbau von Gefängnissen ist sicherlich ein Weg abweichendem Verhalten zu begegnen, allerdings wohl kein präventiver und pädagogischer. Könnte es mit andern Feldern der Devianz als der Kriminalität ähnlich bestellt sein? Wird vieles an Interventionsmöglichkeiten erst dann - kostenintensiv - in Gang gesetzt, wenn das Kind in den Brunnen gefallen ist? Und liegt es nicht nahe, die Schule zu einer Institution der Prävention abweichenden Verhaltens zu gestalten anstatt zu einer der Förderung desselben? Kann die Schule das überhaupt? Und wie sollte eine Schule gestaltet sein, in der eine solche Präventionsarbeit gewährleistet sein könnte? Zudem stellt sich aus der sozialpädagogischen Perspektive heraus die Frage, welchen Beitrag die Sozialpädagogik zur Konzeption und Gestaltung einer solchen Schule zu leisten vermag? Kann dieser Beitrag gar so weit gehen, dass die Rede von einer sozialpädagogischen Schule möglich wäre? Doch auf welcher Tradition baut eine derartige sozialpädagogische Schule auf und worin liegt ihr sozialpädagogisches Charakteristikum?

Im folgenden wird den Ursprüngen einer Verbindung von Schul - und Sozialpädagogik nachgegangen. Dabei wird ersichtlich werden, dass die Vision einer sozialpädagogischen Schule schon auf eine lange, jedoch zeitweise vergessene, Tradition zurückblicken kann. Anschließend wird die präventive Wirkung einer solchen Schule hinsichtlich der Problematiken abweichenden Verhaltens aufgezeigt.

\section{Die sozialpädagogische Schule $^{1}$}

Den Weg zur Beschreibung der sozialpädagogischen Schule bereitet ein historische Besinnung darauf, was unter Sozialpädagogik verstanden werden kann. Gewöhnlich wird Sozialpädagogik heute im Sinne einer außerschulischen Jugendhilfe verstanden. Dies hat seine Gründe, denn unter anderen verortete Klaus Mollenhauer (1974) die Sozialpädagogik als gesellschaftliches Konfliktlösungsmodell einer Jugendhilfe außerhalb der Schule. Während bei der Schulpädagogik der Anlass aller Maßnahmen eine gesellschaftliche, objektive Leistungsanforderung sei, so bei der Sozialpädagogik ein Konflikt, eine Unterscheidung, die heute obsolet zu sein scheint, da die Konfliktbewältigung den Schulalltag vieler Schulen dominiert (PUHR, 2003, S. 90-97). Die berufspolitische Intention zwischen den 1960er und 1980er Jahren, die diese radikale Trennung von Schulpädagogik und Sozialpädagogik begleitet, ist hervorzuheben. Im Rahmen des Ausbaus des Sozialstaats lockte die Verheißung eines professionellen und wissenschaftlichen Aufbruchs, der sich in einem immensen quantitativen Ausbau von Stellen für Sozialarbeiterinnen und Sozialpädagoginnen sowie in einer stetig steigenden Fachlichkeit niederschlug. Im Rausch dieser Jahre justiça no valor de 1,4 bilhões de euros (PFEIFFER, 2005). A construção de prisões é seguramente um caminho para combater comportamentos não-conformados, mas certamente não o é de forma preventiva nem pedagógica. Há outras questões que precisam ser feitas: poderia haver outros campos de semelhança entre os que cometem atos não conformes e criminalidade? Quais são as muitas possibilidades de intervenção acionadas - com altos custos - quando ‘a criança já caiu no poço’? Não seria mais adequado tornar as escolas instituições de prevenção de comportamento desviante ao invés fomentadoras de tal situação? A escola poderia efetivar um trabalho de prevenção? E como deveria ser organizada uma escola que pudesse assegurar um trabalho de prevenção?

Neste contexto, na perspectiva sociopedagógica, coloca-se a questão: qual a contribuição da pedagogia social para a concepção e a realização de uma escola deste tipo? Poderia esta contribuição ser de tal abrangência, que seria possível falar de uma escola sociopedagógica? Mas, sobre qual tradição construir-se-ia tal escola e sobre qual caráter repousaria?

Na seqüência serão examinadas as origens de uma relação entre pedagogia escolar e social. Nisto se evidenciará que a visão de uma escola sociopedagógica pode ser buscada de uma longa, mas temporariamente, esquecida tradição. Subseqüentemente, será apresentado o resultado de um trabalho preventivo de uma escola deste tipo com relação às problemáticas do comportamento não-conformado ou desviante.

\section{A escola sociopedagógica ${ }^{4}$}

O caminho para descrever a escola socio-pedagógica aponta para a reflexão do que pode ser compreendido como pedagogia social. Comumente a pedagogia social é compreendida hoje no sentido da assistência para a juventude extra-escolar. Isto tem seus motivos, pois Klaus Mollenauer (1974), entre outros, define a pedagogia social como profissão para atuar na solução de conflitos sociais em programas de assistência para a juventude fora da escola. Enquanto que na pedagogia escolar o motivo de todas as medidas encontra-se na exigência de resultados sociais e objetivos. Essa diferenciação parece obsoleta já que a resolução de conflitos domina hoje o cotiadiano de muitas escolas (PUHR, 2003, p. 90-97).

Faz-se necessário destacar a intenção políticoprofissional entre os anos 1960 e 1980, que segue essa radical separação entre pedagogia escolar e pedagogia social. O contexto da expansão do Estado Social atraiu a promessa de expansão científica e profissional, que se expressou numa imensa ampliação quantitativa de vagas para assistentes sociais e pedagogos sociais, bem como intensificou o proces- 
gingen andere Traditionslinien der Sozialpädagogik jedoch fast völlig verloren ${ }^{2}$.

Heute jedoch könnten genau diese vor einer Renaissance stehen. Die Sozialpädagogik kann seit ihren Anfängen bei Karl Mager (1820-1858) und Adolph Diesterweg (1790-1866, selber Volksschullehrer und Lehrerausbilder) als das Aufspüren einer pädagogischen Antwort auf die soziale Frage der jeweiligen Epoche verstanden werden. Ein entscheidender Ort für eine solche pädagogische Antwort ist die Schule. In der Epoche Diesterwegs und Magers bestand die soziale Frage vor allem in der Verelendung des Proletariats im Rahmen der Industrialisierung. Es drohte dabei die Gefahr einer Radikalisierung des Politischen und Gesellschaftlichen hin zu totalitären Systemen, entweder einem reaktionär absolutistischen oder einem sozialistischen. Der aufgeheizten Stimmung in dieser Epoche wollte die Sozialpädagogik in dieser Traditionslinie $^{3}$ als eine gemäßigte, dem bürgerlichen Lager verpflichtete Bewegung mit pädagogischen Mitteln begegnen. Das Mittel war eine Bildung des einzelnen Menschen zu einem emanzipierten, aufgeklärten und selbst bestimmten Individuum, das sich aber gleichzeitig dem Gemeinwohl der Gesellschaft gegenüber verpflichtet sah (KONRAD, 1998; MÜLLER, 2002, S. 4-24).

Das Ideal der am demokratischen Gemeinwesen orientierten Bürgerinnen und Bürger ist heute im Rahmen der Debatten um Bürger - und Zivilgesellschaft aktueller denn je. Die heutige soziale Frage ist dabei gekennzeichnet durch die Herausforderungen der Ambivalenzen von Integration und Desintegration ${ }^{4}$. Zur Bewahrung eines demokratischen Gemeinwesens unter diesen Herausforderungen bedarf es einer Erziehung zur Demokratie ${ }^{5}$. Für die pädagogische Antwort auf die soziale Frage von heute ist die Schule dabei weiterhin ein entscheidender Ort.

Aber wie soll die Schule aussehen, in der die soziale Frage eine pädagogische Antwort erhält? Auch hierauf finden wir Antworten in einer historischen Betrachtung der Sozialpädagogik. Franz Michael Konrad verweist auf die enge Verbindung des Begriffs Sozialpädagogik mit einer gewünschten und angestrebten Schulreform in Deutschland zwischen den Jahren 1900 und 1930. Die angestrebte Schulreform zielte unter anderem auf die Schulgeldfreiheit und vor allem die Einrichtung der Einheitsschule (KONRAD, 1998, S. 45-50). Herman Nohl beschreibt diese Einheitsschulbewegung als Ausdruck einer Suche nach der Überwindung der Zerspaltung des Schulwesens, welche für die reine Reproduktion der Klassen der damaligen Zeit verantwortlich gemacht wurde. Im Sinne eines humanistischen Bildungsideals zielte diese Einheitsschulbewegung, so heterogen sie in ihren verschiedenen Ansätzen (sozialistisch, christlich und/ oder nationalistisch bewegt) auch gewesen sein mag, auf eine Bildungseinheit, die den Kindern und so de profissionalização dessas categorias. No êxtase desses anos, outras linhas de tradição da pedagogia social foram completamente perdidas ${ }^{5}$.

Todavia, hoje, exatamente estas podem estar prestes a renascer. A pedagogia social, desde seus iniciantes em Karl Mager (1820-1858) e Adolph Diesterweg (1790-1866, ele mesmo professor de escolas populares e professor de professores), pode ser compreendida como o descobrimento de uma resposta pedagógica para a questão social daquela época. Um lugar decisivo para tal resposta pedagógica seria a escola. Na época de Diesterwegs e Magers, a questão social pousava, sobretudo, na miséria do proletariado no contexto da industrialização. Pairava a ameaça do perigo de uma radicalização do político e do social em direção a sistemas totalitários, que podiam ser absolutistas reacionários ou socialistas. A pedagogia social desta linha de tradição, contrapondo a atmosfera incendiária da época, pretendia abordar o movimento comprometido com os interesses burgueses por meios pedagógicos adequados. $\mathrm{O}$ meio era a formação de cada pessoa para um indivíduo emancipado, esclarecido e auto-determinado, mas que estaria concomitantemente comprometido com o bemestar da sociedade (KONRAD, 1998; MÜLLER, 2002).

$O$ ideal de uma sociedade democrática que orienta os cidadãos e cidadãs é hoje, no contexto dos debates acerca da sociedade civil e cidadania, mais atual do que nunca. A questão social atual é marcada pelos desafios da ambivalência de integração e desintegração ${ }^{6}$. Na a manutenção de um coletivo democrático sob estes desafios, necessita-se uma educação para a democracia ${ }^{7}$. Como resposta pedagógica para a questão social atual, a escola continua sendo um lugar decisivo.

Mas como deve se parecer uma escola, na qual a questão social recebe uma resposta pedagógica? Também para esta encontramos respostas numa apreciação histórica da pedagogia social. Franz Michael Konrad aponta para a estreita relação entre os conceitos da pedagogia social e os da desejada reforma escolar na Alemanha entre os anos 1900 e 1930. A reforma escolar almejava, entre outros, a liberdade financeira das escolas e, sobretudo, a unidade escolar (KONRAD, 1998). Herman Nohl descreve o movimento pela unidade escolar como uma manifestação pela superação da divisão do sistema escolar, por esta ser uma simples reprodução das classes daquele tempo. No sentido de um ideal de formação humanista, este movimento pela unidade escolar, por mais heterogêneos que fossem seus princípios (socialistas, cristãos e/ou nacionalistas), objetivava uma unidade de formação que permitisse, às crianças e aos jovens, de todas as classes e camadas sociais, condições de desenvolvimento individual e a experiência de uma comunidade para 
Jugendlichen aller Klassen und Schichten individuelle Entfaltungsmöglichkeiten und die Erfahrung von einer Gemeinschaft über die Klassenschranken hinweg ermöglichen sollte(NOHL, 1963, zu einer Beschreibung der Bewegung von ihren Anfängen bis 1919 besonders SALLWÜRK, 1920). Der Begriff Sozialpädagogik dient in dieser Bewegung daher dem Vermitteln von zwei Forderungen. Einerseits der Betonung der Gemeinschaft für eine sinnvolle Erziehungs - und Bildungsarbeit. Zweitens dem Ansinnen eines pädagogisch organisiertem und reflektiertem Durchbrechens des Klassen- und Standesdenkens im Sinne eines republikanisch-demokratischen Ideals.

Diese Bewegung gipfelt inhaltlich in den Entwürfen des zurecht als Sozialpädagogen zu bezeichnenden pragmatischen Philosophen John Dewey (1859-1952), ein US-Amerikaner, der sich auf diese Tradition der deutschen Sozialpädagogik stützt (MÜLLER, 2005).

Der Schüler soll dabei vom Objekt des Lehrens zum Subjekt des Lernens werden. Der Unterrichtsstoff wird nicht vorgegeben, sondern Probleme sollen als solche erfahren und als Projekt in der Gruppe gelöst werden. Dewey entschlüsselte den engen Bezug zwischen der Übermittlung sozialer und gesellschaftlicher Kompetenzen und der tätigen Auseinandersetzung mit der Umwelt. Seine oft auf die Parole learning by doing reduzierte Theorie prägte das amerikanische Bildungs - und Schulwesen theoretisch und praktisch entscheidend mit. Sie basiert auf der Einsicht, dass Erziehung und Entwicklung des Menschen nicht auf Übermittlung von Informationen auf rein rationaler Ebene beruhen, sondern auf eigenen Erfahrungsprozessen mit der Umgebung. Ihm ging es darum, das Schulwesen zu reformieren, es einer immer komplexer werdenden Gesellschaft anzupassen. Erziehungsaufgaben, die bis dahin die Großfamilie wahrgenommen hatte, sollte nun die Schule übernehmen. Dewey schwebte eine Schule vor, die zu einer embryonic community werden sollte, die in ihren Grundzügen der Idealfamilie der vorindustriellen Zeit entsprach. Die Schule sollte für die Kinder zu einer Umgebung werden, zu einem Ort, wo das Kind lebt (MÜHREL, M., 2000).

Es wird der Schule dadurch (durch Einführung praktischer Tätigkeiten - Anm. d. Verf.) die Möglichkeit geboten, sich mit dem Leben zu verbinden, des Kindes Heim zu werden, worin es durch ein wohlgeleitetes Leben lernt; anstatt nur ein Ort zu sein, in dem man seine Aufgaben lernt, die eine abstrakte und nur entfernte Verbindung mit irgend einem möglichen Berufe in ferner Zukunft haben. Es wird dadurch der Schule die Möglichkeit geboten, eine Miniaturgemeinschaft, eine embryonische Gesellschaft zu werden (DEWEY, 1905, S.11). além dos limites de classe (NOHL, 1963; SALLWÜRK, 1920). Neste movimento, o termo pedagogia social convém para a difusão de duas exigências. De um lado, a ênfase na comunidade, para um trabalho de educação e formação cheio de sentido. De outro, a exigência de um irromper pedagógico, organizado e refletido do pensar de classe, no sentido de um ideal republicano e democrático.

Este movimento culmina, em termos de conteúdo, nos projetos do filósofo pragmático norteamericano John Dewey (1859-1952), que se apóia na tradição da pedagogia social alemã (MÜLLER, 2005). O aluno, de objeto do ensino, deve tornarse sujeito do aprender. O conteúdo das aulas não é predeterminado, mas problemas devem ser experimentados enquanto tais e resolvidos em forma de projeto grupal. Dewey solucionou a estreita relação entre a transmissão de competências sociais e estudo ativo do meio ambiente. Sua teoria, muitas vezes reduzida ao freqüente ditado learning by doing, cunhou o sistema educacional e escolar norte-americano de maneira decisiva em termos teóricos e práticos. Ela se fundamenta na visão de que a educação e o desenvolvimento da pessoa não se sustentam na transmissão de informações no patamar apenas racional, mas por meio de processos de experiências com o meio, com o contexto. Para ele, tratava-se de reformar o sistema escolar, adequando-a a uma sociedade cada vez mais complexa. Tarefas de educação, que até naquele momento eram realizadas pela família extensa, deviam ser assumidas pela escola. Dewey tinha a idéia de uma escola, que deveria se tornar uma embryonic community, cujos elementos correspondessem aos ideais de família da sociedade pré-industrial. A escola deveria tornar-se para as crianças um contexto, um lugar, no qual pudessem viver (MÜHREL, M.,2000).

\footnotetext{
À escola é colocada a possibilidade, por implantação de atividades práticas - o aprendizado por processo -, de ligar-se com a vida, tornar-se lar da criança, onde ela aprenda por meio de uma vida bem conduzida, ao invés de ser um lugar no qual apenas se aprende as suas tarefas, as quais têm uma relação distante e abstrata com alguma profissão possível num futuro próximo. Por meio deste, é colocada à escola, a possibilidade de se tornar uma comunidade em miniatura, uma sociedade embrionária (DEWEY, 1905, p. 11).
}

Dewey $^{8}$ entendia a educação como procedimento ativo e construtivo, que exige um aprendizado vital e orientado, próximo à ação. Por intermédio da práxis é oferecido um contato direto com a realidade, de forma que poderiam ser realizadas experiências necessárias e pessoais. Decisivo era a apropriação do 
Dewey verstand Bildung als aktiven und aufbauenden Vorgang, der ein handlungsorientiertes und lebensnahes Lernen forderte. Durch die Praxis war ein direkter Kontakt mit der Wirklichkeit gegeben, so dass notwendige und persönliche Erfahrungen gemacht werden konnten. Entscheidend war die Selbstaneignung des Wissens durch den Schüler. Die Eigentätigkeit ist ein wichtiger Bestandteil seiner Pädagogik, da nur durch sie Erfahrungen und Erkenntnisse über die Zusammenhänge der Welt gemacht werden können und sie zur Motivation als Ausgangspunkt für weitere Erfahrungen führt.

Durch Erfahrung lernen heißt das, was wir den Dingen tun, und das, was wir von ihnen erleiden, nach rückwärts und vorwärts miteinander in Verbindung bringen. Bei dieser Sachlage aber wird das Erfahren zu einem Versuchen, zu einem Experiment mit der Welt zum Zwecke ihrer Erkennung. Das sonst bloß passive Erleiden wird zum Belehrtwerden, d.h. zur Erkenntnis des Zusammenhangs der Dinge (DEWEY nach SCHREIBER, 1998, S. 42)

Dewey fertigte Pläne für seine Idealschule an, in denen er die enge Verknüpfung zwischen Theorie und Praxis veranschaulicht. Vorgesehen waren Werkstätten, Laboratorien, Räume für die darstellenden Künste und die Musik, eine Bibliothek und ein Industriemuseum. Bibliothek und Museum sollten die Orte sein, in denen die praktischen Erfahrungen theoretisch ergänzt, gefestigt und vertieft werden sollten, um wiederum in die laufenden Arbeiten der Werkstätten und Laboratorien integriert zu werden. Schüler und Lehrer konnten ihre Erkenntnisse und Erfahrungen bei gemeinsamen Mahlzeiten im großen Speisesaal austauschen und sich gegenseitig zu neuen Aktivitäten ermuntern. In einem Garten sollte den Kindern Gelegenheit geboten werden, das Gedeihen der Pflanzen und des Gemüses zu beobachten, um sich der Herkunft der Nahrungsmittel bewusst zu werden. Das Industriemuseum kam Deweys Vorstellung der inneren Verbindung von Kunst, Wissenschaft und Industrie am nächsten. Seine Aufgabe sollte darin bestehen, eine Verbindung zu anderen Ländern herzustellen, damit Interesse und Verständnis für andere Länder und Völker zu wecken und Vorurteile zu korrigieren. Die Idealschule, wie sie Dewey vorschwebte, wurde nie gebaut. Allerdings versuchte er einige seiner Ideen in seiner VersuchsElementarschule, die der Universität von Chicago angegliedert war und die er 1897 eröffnete, zu verwirklichen (MÜHREL, 2000) ${ }^{6}$.

In diesen Beschreibungen der Reformpädagogik ${ }^{7}$ Deweys sind viele Ideen für ein Reformvorhaben der Schule in den heutigen Debatten nach dem Pisa-Schock in Deutschland wieder zu erkennen. Aus sozialpäda conhecimento pelo aluno. A ação individual é um componente importante de sua pedagogia, já que somente por intermédio desta podem ser realizadas a experiência e o conhecimento sobre as relações no mundo, bem como pode despertar a motivação para outras e novas experiências.

\begin{abstract}
Aprender por meio da experiência significa que nós fazemos às coisas, que nós por elas sofremos, unimos o anterior e o posterior, o adiante. Nesta situação, todavia, a experiência se torna uma tentativa, um experimento com o mundo, com vistas a reconhecê-lo. O dantes sofrimento passivo anterior torna o ser instruído no reconhecimento das relações entre as coisas (DEWEY apud SCHREIBER, 1998, p. 42).
\end{abstract}

Dewey produziu planos para a sua escola ideal, nos quais visualizava estreita relação entre teoria e prática. Estavam previstas oficinas, laboratórios, espaços para a exposição de artes e música, uma biblioteca e um museu da indústria. A biblioteca e o museu deveriam ser lugares nos quais as experiências práticas deveriam ser complementadas, firmadas e aprofundadas, para, de outro lado, serem novamente integradas aos trabalhos desenvolvidos nas oficinas e laboratórios. Alunos e professores poderiam trocar suas descobertas e experiências nas refeições conjuntas no grande refeitório e incentivar-se mutuamente para novas atividades. Em um jardim deveria ser oportunizado às crianças observarem o crescimento de plantas e verduras, a fim de que pudessem conscientizar-se sobre a origem dos mantimentos. Na visão de Dewey, o museu da indústria aproximava intimamente a arte, a ciência e a indústria. Sua tarefa consistia em realizar uma relação com outros países, a fim de despertar o interesse e a compreensão entre países e povos e corrigir preconceitos. A escola ideal, conforme antevista por Dewey, nunca foi construída. Mas ele procurou colocar algumas de suas idéias em prática no Colégio de Aplicação, vinculado à Universidade de Chicago e fundado por ele em 1897 (MÜHREL, 2000) . $^{9}$

Nas descrições da pedagogia de reforma de Dewey, é possível reconhecer muitas idéias do projeto de reforma contido nos debates atuais após o pisa-choque na Alemanha ${ }^{10}$. Na visão sociopedagógica há duas questões a enfatizar. De um lado, a 'sociedade embrionária' ${ }^{11}$ da escola serve como lugar de aprendizado do pensar e do agir democráticos, da educação para a democracia, isto é, da ação enquanto pessoa adulta num sistema democrático, com a aceitação de direitos e responsabilidades. De outro lado, e somente desta forma a democracia é possível, na escola precisam ser consideradas as condições sociais para o aprendizado. A escola não é somente uma entre outras cir- 
gogischer Sicht ist dabei zweierlei zu betonen. Zum einen dient die embryonische Gesellschaft der Schule als Raum des Lernens demokratischen Denkens und Handelns der Erziehung zur Demokratie, d.h. zu einem Handeln als erwachsener Mensch in einem demokratischen Gemeinwesen mit der Übernahme von Rechten und Pflichten. Zum anderen, und nur so kann Demokratie gelingen, müssen die sozialen Bedingungen des Lernens in der Schule berücksichtigt werden. Die Schule ist nicht nur ein Lebensumstand der Schüler neben anderen, sondern sie ist der Ort, wo die Lebensumstände der Schüler artikuliert, berücksichtigt und im emanzipatorischen Sinne im Lernen eingebunden werden müssen. Die Schülerinnen und Schüler bringen über ihre sozialen Hintergründe die heutige soziale Frage der Integration bzw. Desintegration mit in die Schule. In einem sozialpädagogischen Ansatz jeglicher Pädagogik in den Schulen sollte daher eine pädagogische Antwort auf die soziale Frage von Integration und Desintegration gesucht und umgesetzt werden (OPP; UNGER, 2003). Anzumerken ist auch, dass die Neurophysiologie durch ihre Erkenntnisse aus naturwissenschaftlicher Sicht die Reform-sozial-pädagogik in ihren Zielen unterstützt ${ }^{8}$.

2 Die präventive Wirkung der sozialpädagogischen Schule bezüglich abweichenden Verhaltens

Wie stehen die Problematiken von Integration bzw. Desintegration und abweichenden Verhaltens in Relation? Wenn Desintegration eine Bedingung für die Möglichkeit abweichenden Verhaltens ist, so wäre die sozialpädagogische Schule, die ja die soziale Frage von Integration und Desintegration mit dem Ziel der Förderung von Integration und Vermeidung von Desintegration bearbeitet, ein Präventionsmodell bezüglich abweichenden Verhaltens. Die Beziehung der beiden Problematiken sei daher nun genauer betrachtet $^{9}$.

Wilhelm Heitmeyer konstatiert in seiner Einleitung zu dem 1997 erstmals erschienen Sammelband über die Frage Was treibt die Gesellschaft auseinander?: „Desintegration avanciert zu einem Schlüsselbegriff der zukünftigen gesellschaftlichen Entwicklung" (HEITMEYER 1997a, S. 9). In dem im selben Jahr erschienen Parallelband Was hält die Gesellschaft zusammen? stellt er als Pendant zu dieser Ausführung fest: „Die Frage nach der Integrationsfähigkeit cunstâncias da vida dos alunos, mas ela é o lugar no qual essas devem ser articuladas, consideradas e, no sentido emancipatório do aprendizado, inspiradas. Os alunos trazem, por intermédio de sua origem, a atual questão social da integração e/ou desintegração no interior da escola, para a qual qualquer escola deveria estar preparada para transformar e responder (OPP; UNGER, 2003). Há que se considerar também, que a neurofisiologia sustenta os objetivos da reforma sociopedagógica por meio de suas descobertas científicas ${ }^{12}$.

\section{Os resultados da sociopedagogia na pre- venção do comportamento divergente}

Como se relacionam as problemáticas referentes à integração e/ou à desintegração e ao comportamento divergente? Se desintegração é um resultado/ efeito do possível comportamento divergente, então a escola sociopedagógica, que atua nessa questão social com o objetivo de fomentar a integração e evitar a desintegração, é um modelo para a prevenção desse tipo de comportamento. É preciso então refletir com mais precisão sobre a relação entre ambas as problemáticas ${ }^{13}$.

Wilhelm Heitmeyer observa, na introdução de sua obra, acerca da pergunta "O que desintegra/divide a sociedade?", que "A desintegração avança para um conceito chave do desenvolvimento social vindouro" (HEITMEYER, 1997a, p. 9). Num outro livro, publicado no mesmo ano, este autor constata: "A pergunta acerca da capacidade de integração de sociedades modernas tornou-se um tema de discussão pública central como também avançou para o campo científico" (HEITMEYER, 1997b, p. 9). Atualmente, nove anos depois, pode-se aderir a essas afirmações de Heitmeyer. A pergunta acerca da integração e desintegração é uma das, senão a central, questões de nosso tempo. Em que isso é possível de ser ancorado? Para Arnold Schwendtke, integração e desintegração formam basilarmente realidades constitutivas para as mudanças na sociedade, pois integração social fracassada leva a inúmeros conflitos individuais e sociais (SCHWENDTKE, 1995). No tempo presente, colocamse primordialmente dois problemas centrais de integração, conforme bem constatado empiricamente pelo Segundo Relatório de Riqueza e Pobreza no Governo Federal (BUNDESMINISTERIUM ..., 2005):

a) A integração de imigrantes de diferentes ori- 
moderner Gesellschaften ist zu einem zentralen öffentlichen wie wissenschaftlichen Diskussionsthema avanciert" (HEITMEYER, 1997b, S. 9). Heute, neun Jahre später, ist diesen Aussagen Heitmeyers nachdrücklich zuzustimmen. Die Frage von Integration und Desintegration ist eine, wenn nicht die zentrale soziale Frage unserer Zeit. Woran lässt sich dies festmachen? Grundlegend bilden nach Arnold Schwendtke Integration und Desintegration konstitutive Gegebenheiten für den sozialen Wandel in Gesellschaften. Misslingende soziale Integration führt zu einer Vielzahl individueller und sozialer Konflikte (SCHWENDTKE, 1995). In der heutigen Zeit stellen sich dabei vornehmlich zwei im 2. Armuts - und Reichtumsbericht der Bundesregierung aus 2005 empirisch gut belegte Integrationsprobleme (BUNDESMINISTERIUM ..., 2005):

a) Die Integration von Immigranten mit unterschiedlichen Herkunftskulturen. Ein Beispiel hierfür sind mangelnde Bildungschancen von Kindern und Jugendlichen aus Migrantenfamilien. Exemplarisch sei hier das Armutsrisiko bei der Bevölkerung mit Migrationshintergrund genannt.

b) Die innerkulturelle Integration von Personen in den Übergängen der Lebensalter, beispielsweise von der Kindheit zum Jugendalter oder von der Schule zum Beruf. Wiederum exemplarisch mag hierfür stehen die Armut von Familien und Kindern, die unter anderem zu einem erhöhten Risiko gesundheitlicher Probleme bei den Kindern und zu deren Ausgrenzung und mangelndem Schulerfolg schon in der Grundschule führt.

Christian Pfeiffer (1999) beschreibt zwei Formen der Desintegration. Zum einen die emotionale Desintegration, die er als erlebte Nichtzugehörigkeit mit der Erfahrung von emotionaler Ausgrenzung und Nichtakzeptanz beschreibt. Zum anderen die sozioökonomische Desintegration, die sich in einer Ausgrenzung bei dem Zugang zu sozialen Positionen, zu Wohlstand und Entwicklungschancen offenbart.

Aus der Sicht des betroffenen Individuums handelt es sich bei den Erfahrungen beider Arten der Desintegration um dynamische Prozesse der Missachtung ihrer Anerkennungswünsche. Mit Axel Honneth (1992) lassen sich drei Anerkennungsweisen bzw. - ebenen beschreiben - die emotionale Zuwendung, die kognitive Achtung und die soziale Wertschätzung - aus denen heraus im Falle einer gelingenden Anerkennung im Rahmen der reziproken Beziehungen mit anderen Menschen das Individuum entsprechend den Anerkennungsweisen Selbstvertrauen, Selbstachtung und Selbstschätzung entwickelt. Was geschieht jedoch im Falle misslingender Anerkennung? Diese führt zur Erfahrung von Missachtung in den Formen der emotionalen und gens culturais, por serem precárias. As chances de formação de crianças e jovens vindos de famílias de migrantes. Exemplarmente podem ser citados aqui os riscos de pobreza na população com contexto de migração.

b) A integração intercultural de pessoas nas passagens de idade ou ciclo de vida, por exemplo, da infância para a juventude ou da vida escolar para a vida profissional. Mais uma vez, de forma exemplar, está colocada aqui a pobreza de famílias e crianças, entre outros, onde as crianças estão sujeitas a riscos maiores de adoecimento, ocasionando exclusão e aproveitamento escolar precário no ensino fundamental.

Christian Pfeiffer (1999) descreve duas formas de desintegração. Uma, a desintegração emocional, que ele descreve como não-pertencimento vivido, acoplada à experiência da exclusão e não aceitação emocional. Outra, a desintegração socioeconômica, que se manifesta na exclusão de acesso a posições sociais, bem-estar e chances de desenvolvimento.

Na visão dos indivíduos, atingidos pelas duas formas de desintegração, esses são processos dinâmicos de desprezo em relação a seus desejos de reconhecimento. A partir de Axel Honneth (1992 ${ }^{14}$ ), é possível descrever três campos ou formas de reconhecimento, quais sejam, a dedicação emocional, a atenção cognitiva e a valorização social. A partir desses campos, no caso de reconhecimento afirmativo no contexto das relações recíprocas com outras pessoas, o indivíduo desenvolve as respectivas formas de reconhecimento, quais sejam, a autoconfiança, a auto-estima e a autovalorização. Todavia, o que ocorre no caso do não efetivo reconhecimento? Essa situação conduz à experiência de desprezo, nas formas de desintegração emocional e socioeconômica, a qual o indivíduo, para manter o equilíbrio, reage com um comportamento de domínio. Para Honneth, no caso positivo, o indivíduo adentra na luta por reconhecimento, mantendo-se nas normas, valores e regras gerais. Mas essa não é necessariamente a regra geral, porque conflitos individuais gerados por desprezo, conforme Lothar Böhnisch (1999), manifestam comportamento divergente - como a violência - em pessoas que não dispõem de recursos emocionais, cognitivos e sociais suficientes. Também num comportamento deste tipo, no sentido 'negativo', o indivíduo mostra sua aspiração por “[...] capacidade de ação situacional e biográfica e equilíbrio psicossocial em situações e constelações de vida críticas" (BÖHNISCH, 1999, p. 11), mesmo tendo sido desencadeado por meio de experiências de desprezo. Todavia, um comportamento de domínio conduz, de outro lado, a conflitos individuais e sociais, como, por exemplo, espirais crescentes de autodesvalorização com base em desprezo contínuo e desintegração ilimitada (SCHREIBER, 1999). Sob o ponto de vista pedagó- 
sozioökonomischen Desintegration. Das Individuum reagiert darauf mit einem Bewältigungsverhalten zum Ausgleichen dieser Missachtungen. Honneth geht dabei vom positiven Falle aus, dass das Individuum dann in einen Kampf um Anerkennung tritt, bei dem es sich an die allgemeinen Regeln, Werte und Normen hält. Dies muss aber nicht der Fall sein. Denn die individuellen Konflikte durch Missachtung in den Modi der emotionalen wie sozioökonomischen Desintegration werden nach Lothar Böhnisch (1999) bei Menschen, die nicht auf ausreichende emotionale, kognitive und soziale Ressourcen zurückgreifen können, durch abweichendes Verhalten, beispielsweise Gewalt, bewältigt. Auch in einem solchen Bewältigungsverhalten im negativen Falle zeigt sich ein Streben des Individuums nach, ,[...] situativer und biographischer Handlungsfähigkeit und psychosozialer Balance in kritischen Lebenssituationen und konstellationen" (BÖHNISCH, 1999, S. 11), wie sie eben durch Missachtungserfahrungen ausgelöst werden. Doch solches Bewältigungsverhalten führt wiederum zu individuellen und sozialen Konflikten, wie beispielsweise Selbstabwertungsspiralen aufgrund andauernder Missachtungen und anhaltender Desintegration (SCHREIBER, 1999). Unter pädagogischen, politischen und auch ökonomischen Gesichtspunkten sind diese Konflikte dann nur - falls überhaupt - mit großem Aufwand zu bewältigen. Als Beispiel sei hier die Resozialisierung als eine Art der Reintegration im Strafvollzug genannt. Gelingende Integration ist daher die beste Prävention der durch soziale Desintegration/Segregation provozierten individuellen und sozialen Konflikte. Zusammenfassend lässt sich aufzeigen, dass abweichendes Verhalten sich in seinen verschiedenen Formen umso eher ausprägt und manifestiert, ,je größer die Desintegrationsbe-lastungen in unterschiedlichen Teildimensionen der Gesellschaft mit der Folge einer negativen Aner-kennungsbilanz" (ENDRIKAT et al, 2002, S. 40) sind ${ }^{10}$.

In welcher Art und Weise kann die sozialpädagogische Schule bezüglich der Problematik abweichenden Verhaltens präventiv Einfluss nehmen? Einerseits durch die Annahme der sozialen Frage der Integration bzw. Desintegration, indem der soziale Hintergrund der Schülerinnen und Schüler, mit welchem die soziale Frage in die Schule getragen wird, pädagogisch bearbeitet und selber wertgeschätzt wird. Andererseits vermittels einer sozialpädagogisch orientierten Pädagogik in der Förderung der emotionalen, physischen, psychischen und sozialen Ressourcen der Schülerinnen und Schüler, damit diese im Falle von Erfahrungen der Desintegration und Missachtung auf ein positives Bewältigungsverhalten und nicht auf solches abweichenden Verhaltens zurückgreifen. Dies kann jedoch nur in einer Schule erreicht werden, die nicht auf eine Anstalt antisozialen gico, político e econômico, estes conflitos são possíveis de serem solucionados com grande esforço. Como exemplo, pode-se aqui citar a ressocialização como uma forma de reintegração no cumprimento de penas. Por isto, a integração de sucesso é a melhor forma de prevenção de conflitos sociais e individuais, provocados pela desintegração e segregação social.

Em síntese, é possível evidenciar que o comportamento desviante se manifesta e se exprime em suas diversas formas, pois “[...] quanto maior é a carga de desintegração em dimensões parciais diferentes da sociedade maior é conseqüência de um saldo de reconhecimento negativo" (ENDRIKAT et al., 2002, p. 40) ${ }^{15}$.

De que maneira a escola sociopedagógica pode ter influência preventiva na problemática do comportamento divergente? De um lado, por meio da aceitação da questão social de integração e desintegração, na medida em que o contexto social dos alunos é considerado e a questão social é tratada, apreciada e trabalhada de forma pedagógica na escola. De outro lado, por meio de uma pedagogia orientada para o incentivo dos recursos emocionais, físicos, psíquicos e sociais dos alunos, a fim de que estes, em caso de experiências de desintegração e desprezo possam recorrer a comportamentos de superação positivos e não desviantes. Todavia, isto somente pode ser alcançado em escolas que não sejam instituições de repressão de comportamento anti-social, o que por si já evoca o comportamento desviante de alunos e professores (BÖHNISCH, 1999, p. 168-178).

Em resumo, em relação ao risco e à problemática do comportamento desviante, a melhor forma de ação preventiva da escola sociopedagógica está baseada no fortalecimento da autoconfiança, auto-estima e autovalorização de cada aluno individualmente. Isto ocorre por meio de:

a) apropriação do conhecimento e da alegria no êxito do aprendizado;

b) incentivo à cooperação e ao agir democrático, ao invés da repressão de comportamento antisocial,

c) incentivo à participação, e com isto, levar à integração, ao invés do estranhamento, do amedrontar e do isolamento, que levam à desintegração.

Estes são recursos que a escola pedagógica pode dispor para o desenvolvimento de comportamento de superação positivo em alunos expostos a experiências de desintegração e desprezo fora da escola.

\section{Perspectivas}

O ponto temporal para uma mudança no sistema escolar alemão parece em geral muito favorável. A sociedade da República Federal Alemã está, com 
Verdrängungsverhaltens, die selber abweichendes Verhalten bei Schülern und Lehrern hervorruft, reduziert ist (BÖHNISCH, 1999, S. 168-178).

Zusammenfassend lässt sich mit Bezug auf das Risiko und die Problematik abweichenden Verhaltens daher folgende präventive Wirkungsweise der sozialpädagogischen Schule beschreiben:

a) Stärkung des Selbstvertrauens, der Selbstachtung und der Selbstschätzung des einzelnen Schülers. Dies geschieht

b) mittels der Selbstaneignung des Wissens und der Freude am Lernerfolg,

c) der Förderung kooperativen, demokratischen Handelns statt antisozialen Verdrängungsverhaltens,

d) der Förderung der Partizipation - und damit Integration - anstatt Entfremdung, Verängstigung und Vereinzelung - und somit Desintegration.

e) Damit dient sie zudem der Bereitstellung von Ressourcen für die Entwicklung eines positiven Bewältigungsverhaltens der Schülerinnen und Schüler bezüglich Erfahrungen von Desintegration und Missachtung außerhalb der Schule.

\section{Ausblick}

Der Zeitpunkt für einen Wandel des deutschen Schulsystems scheint insgesamt günstig zu sein. Die Gesellschaft der Bundesrepublik ist aufgrund der wissenschaftlichen Erkenntnisse und des öffentlichen, internationalen Drucks verhalten bereit, die Schule zu reformieren. In den kommenden zwanzig Jahren wird sich das Schulwesen in Deutschland wohl grundlegend verändern. Dabei ist eine inhaltliche Ausgestaltung der Schule im Sinne der Sozial-reform-pädagogik Deweys richtungweisend. Lehrerinnen und Lehrer sollten demnach genauso wie Erzieherinnen und Erzieher (Frühpädagoginnen und Frühpädagogen) akademisch gebildete Sozialpädagoginnen bzw. Sozialpädagogen, oder doch zumindest sozialpädagogisch geschult, sein. Zudem ergeben sich für ein zukünftig wünschenswertes multiprofessionelles Team in der Schule für Sozialpädagoginnen und Sozialarbeiterinnen interessante Berufsfelder. Mit Schulsozialarbeit als sozialer Tupfer in Haupt - und Sonderschulen ist es eben nicht getan ${ }^{11}$. Diese sicherlich positiven Ansätze der Schulsozialarbeit gehören ausgebaut in einer umzusetzenden Einheitsschule im Sinne Deweys oder/und nach finnischem Vorbild. Hier werden sich Chancen für die soziale Arbeit insgesamt auftun. Auch die außerschulische Jugendhilfe wird dann gesellschaftlich akzeptabel und durchsetzbar bleiben, wenn sie sich aus der Mitte der Schule als notwendige sozialpädagogische Hilfe für die Familie und den Sozialraum des Schülers erweist, ohne die der base em conhecimentos científicos e pressão pública e internacional, disposta a reformar a Escola. Nos próximos 20 anos o sistema escolar deverá mudar basilarmente na Alemanha. Nesse processo, será determinadora a mudança da orientação interna das escolas no sentido da reforma social-pedagógica defendida por Dewey. Professores deverão possuir formação acadêmica enquanto educadores (educadores infantis), da mesma forma que os pedagogos sociais. Nesse contexto, formar-se-á um interessante campo de atuação profissional, em equipes multiprofissionais na escola, tanto para os pedagogos sociais como para os assistentes sociais. Este processo não mudará a situação dos assistentes sociais que já atuam em escolas especiais e básicas ${ }^{16}$. Certamente os fundamentos positivos do Serviço Social escolar poderão ser dirigidos de forma ampliada à escola unitária, a ser criada, no sentido de Dewey ou no exemplo finlandês. A partir do que poderão ser criadas novas possibilidades de atuação para o Serviço Social. Também a assistência para a juventude no âmbito extra-escolar deverá ser efetivada e aceita socialmente, para que ela, partindo da escola, mostre-se como um necessário auxílio sociopedagógico para famílias e ambiente escolar de alunos, quando o êxito escolar das pessoas jovens for posto em questão. Em contraposição, atualmente, a consideração sobre este contexto extra-escolar é pensado com dificuldades na conjuntura financeira e sociopolítica do país.

Por último, coloca-se uma questão: a pedagogia está aproveitando as chances de ser um ator central no processo de reforma da escola? Alguns pedagogos sociais certamente responderão afirmativamente. Para Müller (2005, p. 259), a pedagogia social deve se dedicar a concepção de escola democrática, "[...] a qual fortaleça o caráter cooperativo do aprendizado e promova a solução de problemas coletivos para além da fixação de uma formação profissional.”

Outros representantes da profissão e de disciplinas científicas se posicionam com dúvidas acerca da possível relação entre pedagogia escolar e pedagogia social. Como, por exemplo, Lothar Böhnisch, Wolfgang Schröer e Hans Thiersch que não conseguem visualizar a possibilidade desta nova determinação para o pensar ${ }^{17}$ sociopedagógico. Assim, eles afirmam: " [...] o Serviço Social procura relacionar acesso à justiça com auxílio para a superação de situações de vida [...]" (BÖHNISCH; SCHRÖER; THIERSCH, 2005, p. 258). Mas vale indagar se não é justamente a escola o espaço-lugar sociopedagógico privilegiado, tendo em vista o acesso à justiça e o auxílio para a superação de situações de vida, para o aqui exposto sentido de uma 'educação para a democracia'? Os autores acima $(2005$, p. 264) ainda afirmam: “[...] sobretudo, é preciso, na perspectiva da concepção de superação de situações de vida, 
Bildungserfolg des jungen Menschen in Frage gestellt ist. Die Behauptung außerhalb jeglichen Schulzusammenhangs wird sich in der derzeitigen finanz - wie sozialpolitischen Situation dagegen äußerst schwierig gestalten.

Es stellt sich nun abschließend eine Frage: Wird die Sozialpädagogik ihre Chancen wahrnehmen und ein Akteur des Reformprozesses der Schulen sein? Einige Vertreter der Sozialpädagogik werden dies bejahen. So hat sich nach Müller die Sozialpädagogik einer demokratischen Schulkonzeption zu widmen, „welche den kooperativen Charakter gemeinsamen Lernens und Problemlösens jenseits einer Fixierung auf berufliche Ausbildung stärkt" (MÜLLER, 2005, S. 259). Andere Vertreter der Profession und wissenschaftlichen Disziplin halten sich bezüglich einer solchen Verbindung von Sozial - und Schulpädagogik eher bedeckt. Hierzu gehören z.B. Lothar Böhnisch, Wolfgang Schröer und Hans Thiersch, die in ihrer Neubestimmung sozialpädagogischen Denkens ${ }^{12}$ diese Möglichkeit ausblenden. Dabei liefern sie selbst Argumente, die auch für eine solche Verbindung sprechen. So führen sie aus: „Soziale Arbeit versucht Zugangsgerechtigkeit mit lebensweltlichen Bewältigungshilfen [...] zu verbinden" (BÖHNISCH; SCHRÖER; THIERSCH, 2005, S. 258). Ist nicht gerade die Schule im dargestellten Sinne der „Erziehung zur Demokratie" in diesem Blickwinkel von Zugangsgerechtigkeit und lebensweltlichen Bewältigungshilfen ein ausgezeichneter, wenn nicht der ausgezeichnete sozialpädagogische Ort? Die Autoren führen weiter fort: „Vor allem aber brauchen aus der Sicht des Lebenswelt- und Bewältigungskonzeptes die Adressatinnen und Adressaten funktionale Äquivalente, in denen sie [...] Selbstwert, Anerkennung und Selbstwirksamkeit erlangen können" (Ebenda, 264). Die sozialpädagogische Schule wäre genau ein solches funktionales Äquivalent, ein solcher funktionaler Gegenwert und Ausgleich zur Wirklichkeit vieler Kinder und Jugendlicher, die durch Missachtung in all ihren gravierenden Formen (HONNETH, 1992) und emotionale wie sozioökonomische Desintegration (PFEIFFER, 1999) geprägt ist.

Erste ermunternde Ergebnisse einer solchen sozialpädagogischen Ausrichtung der Schule zeigt ein Projekt im Bundesland Niedersachsen, in welchem über bewegungs-und theaterpädagogische Zugänge Schülerinnen und Schüler der Grund -, Haupt - und Sonderschulen Selbstbewusstsein und Selbstkompetenz erlangen und ihr soziales Verhalten trainieren. Dadurch gelangen sie in die Lage, ihre Lebenssituation allgemein wie ihre schulische Situation im Besonderen reflexiv wahrzunehmen. Dies wiederum fördert positives Bewältigungsverhalten in Konflikten. equivalência funcional dos endereçados, a fim de que possam alcançar auto-estima, reconhecimento e autoefetivação."

A escola sociopedagógica seguramente seria este 'equivalente funcional', o análogo e a compensação para a realidade de muitas crianças e jovens, marcados por todas as formas desprezo (HONNETH, 1992,) e por desintegração emocional e socioeconômica (PFEIFFER, 1999).

Os primeiros resultados animadores de uma perspectiva sociopedagógica nesta direção mostram um projeto no estado federal da Baixa Saxônia, no qual, através de iniciativas de movimento e teatro pedagógicos, os alunos de escolas elementares e especiais alcançam autoconsciência, autoconfiança e autocompetência e exercitam seu comportamento social. Por meio desse processo eles atingem as condições de perceber e refletir as situações de vida de forma geral, bem como as situações escolares de maneira especial. Esse processo também estimula o domínio comportamental positivo, em caso de conflitos.

\section{Notas}

1 Nota da Tradução: No texto original o termoutilizadoé Devianz, cujo significadoéo comportamento divergente da norma regular, ou seja, o irregular, o divergente, o não conforme. É o comportamento individual ou grupal visto como não desejado ou não correto tendo em vista normas e valores de uma sociedade. Vale esclarecer que este termo tem o sentido do desviante, aquele a quem a atuação de profissionais deve estar especialmente voltada, para que, justamente com a intervenção especializada se atinja novamente o patamar desejado de desenvolvimento social. No Brasil, este termo tem um sentido essencialmente negativo, pois denota a inadaptação do indivíduo ao meio social. O desvio primário se refere a alguma atitude ou ação contra as normas instituídas, podendo ser tanto na escola como na família. Já o desvio secundário consiste na formalização do desvio primário nas instâncias de justiça e controle social (polícia, juizado da juventude, entre outros), sendo que esta intermediação desencadeia comportamento correspondente por parte do adolescente.

2 Nota da tradução: Mobbing, termo usado para situações de humilhação a que são submetidos os alunos, principalmente por parte dos colegas.

3 Nota da tradução: O Hartz IV é o nome popular do novo Programa de Auxílio Desemprego, que entrou em vigor a partir de 01.01.2005, sendo a junção de dois programas anteriores, $\mathrm{o}$ Auxílio Desemprego e oAuxílio Social. Os críticos destacam que este programa significou perdas financeiras para os demandantes, já que alguns auxílios (como por exemplo, os auxílios para aquisição de roupas, móveis e utensílios domésticos, material escolar) podem ser requisitados apenas umaúnica vez. 


\section{Literatur}

BÖHNISCH, L. Abweichendes Verhalten: Eine pädagogisch-soziologische Einführung. Weinheim u. München, 1999.

;) SCHRÖER, W.; THIERSCH, H. Sozialpädagogisches Denken: Wege zu einer Neubestimmung. Weinheim, 2005.

\section{BUNDESMINISTERIUM FÜR GESUNDHEIT UND} SOZIALE SICHERUNG. (Hrsg.). Lebenslagen in Deutschland: Der 2. Armuts - und Reichtumsbericht der Bundesregierung. Berlin, 2005.

DEWEY, J. Schule und öffentliches Leben. Berlin, 1905.

DUNGS, S. Anerkennungsmodelle des Anderen. Sozialphilosophische Reflexionen und ihre Bedeutung für die Soziale Arbeit. In: MÜHREL, Eric. Quo vadis Soziale Arbeit? Auf dem Wege zu grundlegenden Orientierungen. Essen, 2006, S. 83-104.

ENDRIKAT, K. et al. Soziale Desintegration. Die riskanten Folgen negativer Anerkennungsbilanzen. In: HEITMEYER, W. (Hrsg.). Deutsche Zustände. Folge I. Frankfurt/M., 2002, S. 37-58.

HEITMEYER, W. Auf dem Weg in eine desintegrierte Gesellschaft. In: DERS. (Hrsg.). Was treibt die Gesellschaft auseinander? Bundesrepublik Deutschland: Auf dem Weg von der Konsens- zur Konfliktgesellschaft. Frankfurt a.M., 1997a, S. 9-28.

Sind individualisierte und ethnisch-kulturell vielfältige Gesellschaften noch integrierbar?. In: DERS. Was hält die Gesellschaft zusammen? Bundesrepublik Deutschland: Auf dem Weg von der Konsens- zur Konfliktgesellschaft. Frankfurt a.M., 1997b, S. 9-22.

HOMFELD, H.-G. et al. Für eine sozialpädagogische Schule. München, 1977.

HONNETH, A. Kampf um Anerkennung. Zur moralischen Grammatik sozialer Konflikte. Frankfurt a.M., 1992.

KONRAD, F.-M. Sozialpädagogik. Begriffsgeschichtliche Annäherungen - von Adolph Diesterweg bis Gertrud Bäumer. In: MERTEN, R. (Hrsg.). Sozialarbeit, Sozialpädagogik, Soziale Arbeit. Begriffsbestimmungen in einem unübersichtlichen Feld. Freiburg i.Br, 1998, S. 31-62

MOLLENHAUER, K. Einführung in die Sozialpädagogik: Probleme und Begriffe der Jugendhilfe. Weinheim, 1974.

MÜHREL, Eric. Sozialpädagogik macht Schule. Eine alte richtungweisende Zukunft. Sozialmagazin, v. 4, n. 5, 2005.
4 Também conformeMührel(2005).

5 Sobre a temática da relacao entre escola e pedagogia social nesta época, ver Homfeld (1977). Este autor afirma a idéia de uma escola sociopedagógica.

6 Sobre este tema, ver item 1 deste artigo.

7 Acerca da educação para a democracia no contexto da pedagogia social, ver Müller(2005).

8 Nota da tradução: Entre as principais obras pedagógicas de Dewey estão The School and Society (Escola e sociedade) e Experience and Education (Experiência e educação), não disponíveis em língua portuguesa e Democracy and Education, disponível em português, com o título Democracia e educação, traduzida por Godofredo Rangel e Anísio Teixeira e publicada em São Paulo pela editora Nacional, em 1959 .

9 As idéias de Dewey podem ser encontradas, entre outros, numa descrição da escola reformada na revista Spiegel Special dedez. 1997.

10 Nota da tradução: O pisa-choque se refere aos impactos com os resultados do Programme for International Student Assessment, coordenada pela OCDE em seus países associados nos anos 2000, 2003 e 2006. Tem o objetivo de avaliar a competência para a leitura, os conhecimentos nas ciências naturais e matemáticas, com vistas a construção de indicadores para informar os países membros sobre as fragilidades e os pontos fortes de seus sistemas educacionais. Os resultados do ano 2000 foram chocantes para a sociedade alemã, desembocando em intensos debates no âmbito político e a adoção de medidas para qualificar o sistema educacional no país.

11 Nota da tradução: No original grifado, a partir de Dewey, embryonische Gesellschaft.

12 Como exemplo, podem aqui ser citados os escritos do pesquisador de cérebro, neurobiólogo e pedagogo Gerald Hüters.

13 Sobre o parágrafo seguinte ver também Mührel(2006a).

14 A obra de Axel Honneth aqui referenciada está disponível no Brasil, com o título Luta por reconhecimento: a gramática moral dos conflitos sociais, traduzida por Luiz Repa. Foi publicada em São Paulo pela Editora 34, em 2003.

15 A relação de reciprocidade entre desintegração e desprezo é aprofundada por Dungs (2006).

16 Isso no caso de contratos de trabalho regulares com profissionais firmados, enão como o planejado, já ocorrendo em alguns municípios, nos quais os trabalhos do assistente 
. Sozialpädagogik und gesellschaftliche Partizipation. Pädagogisch reflektierte und organisierte Sozialisation. Soziale Arbeit. Nr. 2, S. 100-104, 2006 a.

. (Hrsg.). Quo vadis Soziale Arbeit? Auf dem Wege zu grundlegenden Orientierungen. Essen, 2006b.

MÜHREL, Michaela. Kinder-Jungendmuseen im Blickfeld der pädagogisch orientierten Kunsttherapie. Unveröffentlichte Diplomarbeit an der Universität zu Köln, Heilpädagogische Fakultät, 2000.

MÜLLER, C. Wir alle sind Aristen... weil Bürger. In: ANDRESEN, S.; TRÖHLER, D. (Hrsg.). Gesellschaftlicher Wandel und Pädagogik: Studien zur historischen Pädagogik. Zürich, 2002, S. 14-24.

. Sozialpädagogik als Erziehung zur Demokratie: Ein problemgeschichtlicher Theorieentwurf. Bad Heilbrunn, 2005.

NOHL, H. Die pädagogische Bewegung in Deutschland und ihre Theorie. Frankfurt a.M., 1963.

OPP, G.; UNGER, N. Begriffliche Grundlagen. In: OPP, G. (Hrsg.). Arbeitsbuch schulische Erziehungshilfe. Bad Heilbrunn, 2003, S. 43-64.

PFEIFFER, C. Weniger Verbrecher, mehr Panikmache. Die Zeit, n. 23, S. 9, 2005.

. et al. Innerfamiliäre Gewalt gegen Kinder und Jugendliche und ihre Auswirkungen. KfN Forschungsberichte: Hannover, n. 80, 1999.

PUHR, K. Schule zur Erziehungshilfe als lernende Organisation. In: OPP, G. (Hrsg.). Arbeitsbuch schulische Erziehungshilfe. Bad Heilbrunn, 2003, S. 65-107.

SALLWÜRK, V. E. Die deutsche Einheitsschule und ihre pädagogische Bedeutung. Langensalza, 1920.

SCHREIBER, Werner, Bildungskonzepte und Bildungswiderstände mit psycho-sozial belasteter Klientel. Neue Praxis, n. 5, S. 457-470, 1999.

SCHREIBER, Ursula. Kindermuseen in Deutschland. Grundlagen, Konzepte, Praxisformen. Unna, 1998.

SCHWENDTKE, A. A. Integration. In: DERS. (Hrsg.). Wörterbuch der Sozialarbeit und Sozialpädagogik. Wiesbaden, 1995, S.131.

SPIEGEL SPECIAL. International Edition, Hamburg, n. 12, 1997. social escolar são desenvolvidos por voluntários e empregos de um euro.

17 Acerca da procura por uma nova determinação ver Mührel (2006b).

\section{Eric Mührel}

Doutor em Filosofia pela Universidade de Essen Pedagogo e Assistente Social

Professor titular na Escola Técnica Superior Oldenburg/Ostfriesland/Wilhelmshaven

Decano do Instituto de Sistemas Sociais no Campus Emden (Alemanha).

\section{Endereço}

Fachhochschule Oldenburg/Ostfriesland/Wilhelmshaven

Studienort Emden, Constantianplatz 4, Sala G-219

D-26723 Emden - Deutschland 